\begin{tabular}{c|c|c|} 
SELECCIONES MATEMÁTICAS \\
Universidad Nacional de Trujillo \\
ISSN: $2411-1783$ (Online) \\
2020; Vol. $7(1): 1-9$.
\end{tabular}

Un modelo matemático-epidemiológico del control de la obesidad

\title{
A mathematical-epidemiological model of obesity control
}

Roxana López Cruz ${ }^{\mathbb{D}}$

Received, Feb. 22, 2020

Accepted, Apr. 30, 2020

How to cite this article:

López R. Un modelo matemático-epidemiologico del control de la obesidad. Selecciones Matemáticas. 2020;7(1):1-9. http://dx.doi.org/10.17268/sel.mat.2020.01.01

\begin{abstract}
Resumen
En este trabajo, se investiga la estabilidad de dos modelos epidemiologicos de la obesidad. El primer modelo solo admite la influencia social, es decir el ambiente en que se desplazan los individuos, el segundo modelo le agrega control para reducir la obesidad y el exceso de peso. El punto de equilibrio libre de obesidad es local y asintóticamente estable si $\mathcal{R},<1$ donde $\mathcal{R}$, es una tasa básica de reemplazo. Para el modelo con control, se muestra la estabilidad del mismo y se aprecia numericamente que acelera la disminución de individuos con obesidad.
\end{abstract}

Palabras clave. Ecuación diferencial ordinaria, Obesidad como epidemia, Estabilidad, simulación.

\begin{abstract}
In this work, we analyze the stability of two epidemiological models of obesity. The first model only admits social influence, that is, the environment in which individuals live, the second model adds control to reduce obesity and excess weight. The obesity-free equilibrium point is local and asymptotically stable if $\mathcal{R},<1$ where $\mathcal{R}$, is a basic replacement rate. For the model with control, its stability is shown and it is appreciated numerically that it accelerates the decrease of obese individuals.
\end{abstract}

Keywords . Ordinary differential equation, Obesity as epidemic, Stability, Simulation.

1. Introducción. La obesidad es una enfermedad crónica que está siendo considerada como epidemia, debido a su alto crecimiento en el número de personas que la adquieren (sin considerar distinción en grupos poblacionales). La implicancia de su evolución influye drásticamente en otras enfermedades como la diabetes entre otras, según los datos de los principales organismos mundiales de la salud como la Organización Mundial de la Salud (OMS) o PanAmerican Health Organization (PAHO). El incremento de las personas que adquieren malos hábitos en su alimentación o el sedentarismo son algunos de los motivos para que las poblaciones tiendan a ser obesas, debido a ese aumento los expertos consideran a la obesidad como una epidemia.

En este trabajo desarrollaremos modelos matemáticos que simulen la evolución temporal de la obesidad como proceso epidemiológico. Y como resultado, pretendemos visualizar la evolución de la obesidad en nuestra realidad lo que permitirá hacer proyecciones que colaboren con los organismos de prevención de enfermedades.

En el Perú, un problema de salud que compromete a otras, es la propagación de la población de obesos, es por ello que la Obesidad es una de las enfermedades en las cuales se debe tener mucho control. Las instituciones encargadas de ese análisis y control son los centros de estudios en salud como el Instituto Nacional de Salud (INS), el Control de Enfermedades Epidemiológicas (CDC) dependientes del Ministerio de Salud y las instituciones con centros de investigación en salud como lo son las universidades con carreras de Medicina y/o Salud Pública. Todas estas centran sus investigaciones en el análisis de modelos estadísticos, que no dejan de ser importantes pero si colaterales de nuevas propuestas como lo son los modelos matemáticos.

\footnotetext{
*Instituto de Investigación Centífica (IDIC), Universidad de Lima, Lima, Perú (rlopez c@ulima.edu •pe).
} 
Esto motiva que en este trabajo se proponga, analize e interprete los resultados por simulación del (los) modelos(s) propuestos(s) para la proyección del comportamiento a lo largo del tiempo de la propagación de la obesidad. Hay que enfatizar que un modelo es como un mapa, trata de reflejar la gran mayoría de aspectos que caracterizan a lo que quiere modelar; es por ello que los modelos evolucionan con mayor complejidad según como se van planteando desde lo mas básico.

Los modelos a estudiar nos permitirán mostrar los diversos escenarios de predicción posibles de la obesidad en varias poblaciones epidemiológicas y la posibilidad de ver los resultados al colocar un control con significado médico y que quede expresado matemáticamente. La divulgación de esta alternativa de análisis de la obesidad como epidemia permitirá obtener una alternativa más de investigación para una toma de decisiones de precisión.

En la literatura universal sobre modelamiento matemático de la obesidad, existen dos aspectos de su estudio, uno desde el punto de vista epidemiológico ya que debido a malos hábitos en la alimentación o actividad física, estos han sido factores de propagación en las poblaciones diversas de esta enfermedad crónica, la obesidad (modelo basado en la población) como en [1], [2], [3] y el otro punto de vista es fisiológico en cuanto al tipo de alimento que se consume (modelo basado en el individuo) [3].

Aquí proponemos modelos que nosotros planteamos a partir de otros modelos que perturbamos según algún nuevo aspecto que consideraremos y la sugerencia es el control de la obesidad traducido en dietas u otras razones de reducción de la población con sobrepeso u obeso. También hay aspectos genéticos de la obesidad que podrán ser incorporados según el avance de la investigación.

2. Modelo matemático básico y preliminares. Para la construcción de este modelo, consideraremos tres subpoblaciones de la población total, individuos con peso normal $N(t)$, individuos con sobrepeso $S(t)$ y aquellos individuos obesos $\mathrm{O}(\mathrm{t})$. También vamos a considerar algunas suposiciones:

- La población total es homogénea, es decir tiene condiciones de salud similares.

- Las personas pasan a las diferentes etapas de peso no normal por el consumo excesivo de comida no sana (harinas, frituras, bebidas gaseosas).

Las transiciones entre estas tres subpoblaciones se suceden de la siguiente manera,

- Una persona de peso normal $\mathrm{N}(\mathrm{t})$ que empieza a excederse en comida no sana inicia su transición a persona con sobrepeso $\mathrm{S}(\mathrm{t})$,

- Una persona con sobrepeso $\mathrm{S}(\mathrm{t})$ que continua con su exceso de comida no sana, pasa a ser persona con obesidad $\mathrm{O}(\mathrm{t})$,

- Las transiciones entre las diferentes subpoblaciones se dan en proporción a las poblaciones de salida, salvo en el caso de causa de que un individuo con peso normal pase a las etapas de sobrepeso $\mathrm{u}$ obesidad, pues estas se relacionan a la influencia social sobre el individuo como por ejemplo las costumbres alimenticias de la familia, en cuyo caso estos cambios se dan en proporción a la influencia de las otras poblaciones (punto de vista epidemiológico), es así como veremos que esta transición viene modelada por el término $\frac{[\alpha S(t)+\beta O(t)] N(t)}{P(t)}$

- Los parámetros de transición son constantes, esto será de mucha utilidad al utilizar el modelo en etapas cortas de edad.

Con todas estas consideraciones, el diagrama de flujo del modelo es

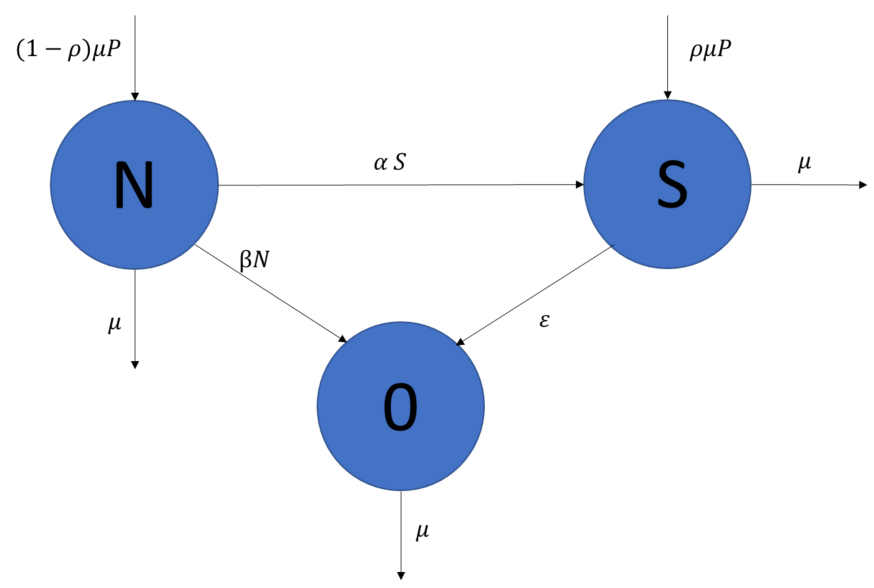

Figura 2.1: Modelo sin control de la obesidad (2.1) 
$\mathrm{Y}$ en ecuaciones diferenciales ordinarias no lineales viene dado por

$$
\begin{aligned}
\frac{d N(t)}{d t} & =(1-\rho) \mu P(t)-\alpha \frac{S(t)}{P(t)} N(t)-\beta \frac{O(t)}{P(t)} N(t)-\mu N(t) \\
\frac{d S(t)}{d t} & =\rho \mu P(t)+\alpha \frac{S(t)}{P(t)} N(t)-(\epsilon+\mu) S(t) \\
\frac{d O(t)}{d t} & =\beta \frac{O(t)}{P(t)} N(t)+\epsilon S(t)-\mu O(t)
\end{aligned}
$$

donde las variables para el modelo básico vienen dadas en el Tabla 2.1

\begin{tabular}{|c|l|}
\hline Variables & Significado \\
\hline$N(t)$ & Población de no obesos en cualquier momento t (personas con IMC menor de 24,9) \\
\hline$S(t)$ & $\begin{array}{l}\text { Población de no obesos con sobrepeso en cualquier momento } \mathrm{t} \text { (personas que empiezan a } \\
\text { ingerir calorías, es decir con IMC entre 25 y 29,9) }\end{array}$ \\
\hline$O(t)$ & Población de obesos en cualquier momento t (personas con IMC mayor que 30) \\
\hline$P(t)$ & Población total en cualquier instante $t, P(t)=N(t)+S(t)+O(t)$ \\
\hline
\end{tabular}

Tabla 2.1: Significados de la variables del sistema (2.1)

\begin{tabular}{|c|l|}
\hline Parámetros & Significados \\
\hline$\rho$ & Probabilidad de que el recién nacido nazca con sobrepeso (se asume que no se nace obeso) \\
\hline$\mu$ & Tasa de nacimiento o muerte natural \\
\hline$\alpha$ & Tasa a la cual un individuo con peso normal empieza a ingerir calorías por la influencia de \\
& un ambiente social de gente con sobrepeso y pasa a gente con sobrepeso \\
\hline$\beta$ & Tasa a la cual un individuo con peso normal empieza a ingerir calorías por la influencia de \\
& un ambiente social de gente obesa y pasa a gente obesa \\
\hline$\epsilon$ & Tasa a la cual un individuo con sobrepeso se convierte en obeso \\
\hline
\end{tabular}

Tabla 2.2: Significados de los parametros del sistema (2.1)

2.1. Reducción del sistema. En el sistema (2.1), observamos que la población total $P(t)$ es constante, dado que $\frac{d P}{d t}=\frac{d(N+S+O)}{d t}=0$, donde $P(t)=P($ cte $)$, podemos escribir $N(t)=P-(S(t)+O(t))$. En consecuencia, el sistema (2.1) se reduce al estudio de un sistema bidimensional dado por

$$
\begin{aligned}
\frac{d S}{d t} & =\rho \mu P(t)+\alpha \frac{S(t)}{P(T)}[P-(S(t)+O(t))]-(\epsilon+\mu) S(t) \\
\frac{d O}{d t} & =\beta \frac{O(t)}{P(t)}[P-(S(t)+O(t))]+\epsilon S(t)-\mu O(t)
\end{aligned}
$$

2.2. Normalización del sistema. Normalizando el sistema (2.2), consideramos las subpoblaciones $s(t)=S(t) P$ у $o(t)=O(t) P$, debido a que la población total es constante, sin perdida de generalidad, se obtiene

$$
\frac{d s}{d t}=\rho \mu+\alpha s[1-(s+o)]-(\epsilon+\mu) s
$$

$$
\frac{d o}{d t}=\beta o[1-(s+o)]+\epsilon s-\mu o
$$


2.3. Análisis del modelo. Existencia de las soluciones

Teorema 1. Para todo $s_{0}, o_{0}>0$, existen $s, o:[0, \infty) \longrightarrow[0, \infty)$ que resuelven el sistema (2.3) con condiciones iniciales $s=s_{0}, o=o_{0}$.

Demostración: La existencia de las soluciones está garantizada por el teorema de Peano.

Teorema 2. Todas las soluciones del sistema (2.3) son acotadas y positivas.

Demostración: El conjunto de soluciones del sistema (2.3) se prueba que son acotadas y positivas con la condición de conjunto positivamente invariante; en el sentido que si las condiciones iniciales del sistema son acotadas y no negativas (región invariante), las soluciones permanecen en dicha región en toda su definición.

2.4. Punto de equilibrio libre de obesidad y tasas básicas de reemplazo. Con (2.1) ya reducido y normalizado al sistema (2.3), buscamos la solución que represente la situación de que una población quede libre de infección, a este se le llama punto de equilibrio libre de obesidad.

Para hallar los puntos de equilibrio, resolvemos el sistema algebraico, indicado por las isoclinas

$$
\begin{aligned}
\rho \mu+\alpha s[1-(s+o)]-(\epsilon+\mu) s & =0 \\
\beta o[1-(s+o)]+\epsilon s-\mu o & =0
\end{aligned}
$$

Teorema 3. Si $\rho=0$, los puntos críticos se dividen en tres casos:

1. El punto libre de sobrepeso y obesidad $(s, o)=(0,0)$ existe si $\alpha=\epsilon+\mu$

2. El punto libre de obesidad $(s, o)=\left(s^{*}, 0\right)$ existe si $\alpha>\epsilon+\mu$.

3. El punto persistencia de sobrepeso y obesidad $(s, o)=\left(s^{* *}, o^{* *}\right)$ existe si $(\epsilon+\mu-\alpha)(\beta-\alpha)>0$, donde

$$
s^{* *}=(\alpha-\epsilon-\mu)\left(\frac{\mu(\beta-\alpha)+\epsilon \beta}{(\epsilon+\mu)(\beta-\alpha)}\right)
$$

$y$

$$
o^{* *}=\frac{\epsilon(\epsilon+\mu-\alpha)}{(\epsilon+\mu)(\beta-\alpha)}
$$

Las tasas básicas de reemplazo son aquellos parámetros de salida que determinan la ocurrencia o no de la eliminación de las poblaciones de individuos con sobrepeso y obesidad. Para su cálculo utilizamos la matriz de la próxima generación.

Teorema 4. Si $\rho=0$, el punto de equilibrio libre de sobrepeso y obesidad es local y asintóticamente estable si $\mathcal{R}=\frac{\mu}{\beta}<1$, es inestable si $\mathcal{R}=\frac{\mu}{\beta}>1$.

Demostración: Al aplicar la matriz de la próxima generación, los autovalores de la matriz de la próxima generación correspondiente son $\lambda_{1}=\frac{\alpha}{\epsilon+\mu}$ y $\lambda_{2}=\frac{\beta}{\mu}$.

El punto de equilibrio libre de sobrepeso y obesidad existe si $\alpha=\epsilon+\mu$, luego $\lambda_{1}=1$. En consecuencia, por el Teorema 2 de la Ref. 11, tenemos que si $\mathcal{R}=\frac{\mu}{\beta}<1$, el punto $(s, o)=(0,0)$ es local y asintóticamente estable y si $\mathcal{R}=\frac{\mu}{\beta}>1$, dicho punto es inestable.

2.5. Simulaciones. Aquí una simulación del modelo de la obesidad dado por (2.1), con condiciones iniciales de data del año $2005(t=0)$ hasta el año donde los valores de las condiciones iniciales vienen dados en la Tabla 2.3

\begin{tabular}{|c|c|c|c|c|}
\hline Notación CI & $N(0)$ & $S(0)$ & $O(0)$ & $P(0)$ \\
\hline Valor & 100 & 35 & 25 & 160 \\
\hline
\end{tabular}

Tabla 2.3: Valores de las condiciones iniciales (CI) del sistema (2.1)

Se puede apreciar de la simulación del modelo (2.1), que ante una población de no obesos de 100 , 35 de los que exhiben sobrepeso y 25 obesos, si las tasas de conversión a las subpoblaciones en riesgo son pequeñas, tendremos el control sobre la obesidad. Nuestro propósito será demostrar teóricamente esta situación. 


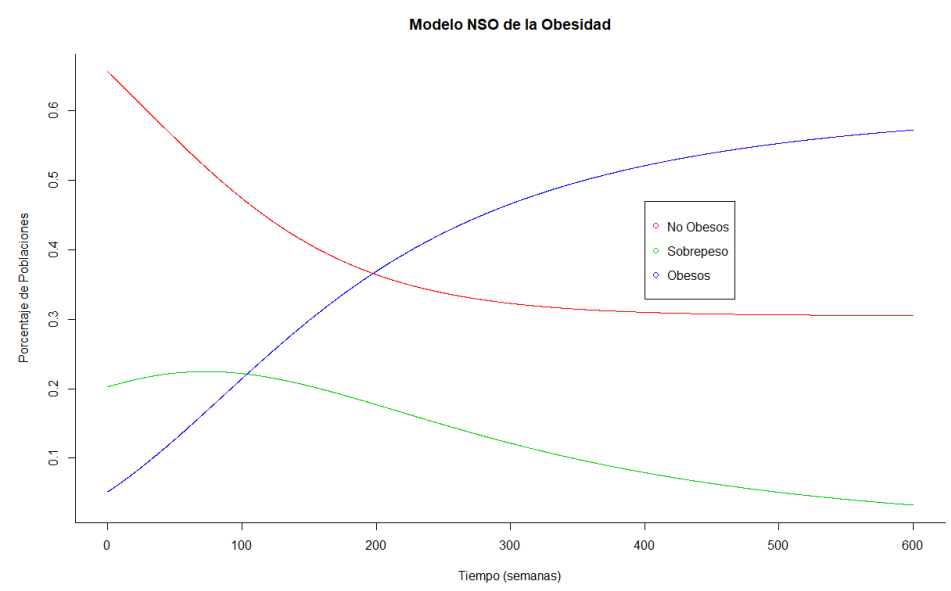

Figura 2.2: Modelo sin control de la obesidad (2.1)

3. Modelo con control de la obesidad. Se plantea un modelo epidemiológico para la obesidad considerando tratamiento traducido en dieta o actividad física. Para la construcción de este modelo, consideraremos igual que con el modelo anterior, tres subpoblaciones de la población total, individuos con peso normal $N(t)$, individuos con sobrepeso $S(t)$ y aquellos individuos obesos $O(t)$. También vamos a considerar las suposiciones:

- la población total es homogénea (es decir tiene condiciones de salud similares).

- Las personas pasan a las diferentes etapas de peso no normal por el consumo excesivo de comida no sana (harinas, frituras, bebidas gaseosas).

- El individuo puede empezar una dieta en cualquiera de las etapas $S(t)$ o $O(t)$.

Las transiciones entre estas tres subpoblaciones se suceden igual que con el modelo (1), solo que aquí consideraremos la etapa de control de la obesidad Con todas estas consideraciones el modelo tiene el diagrama de flujo

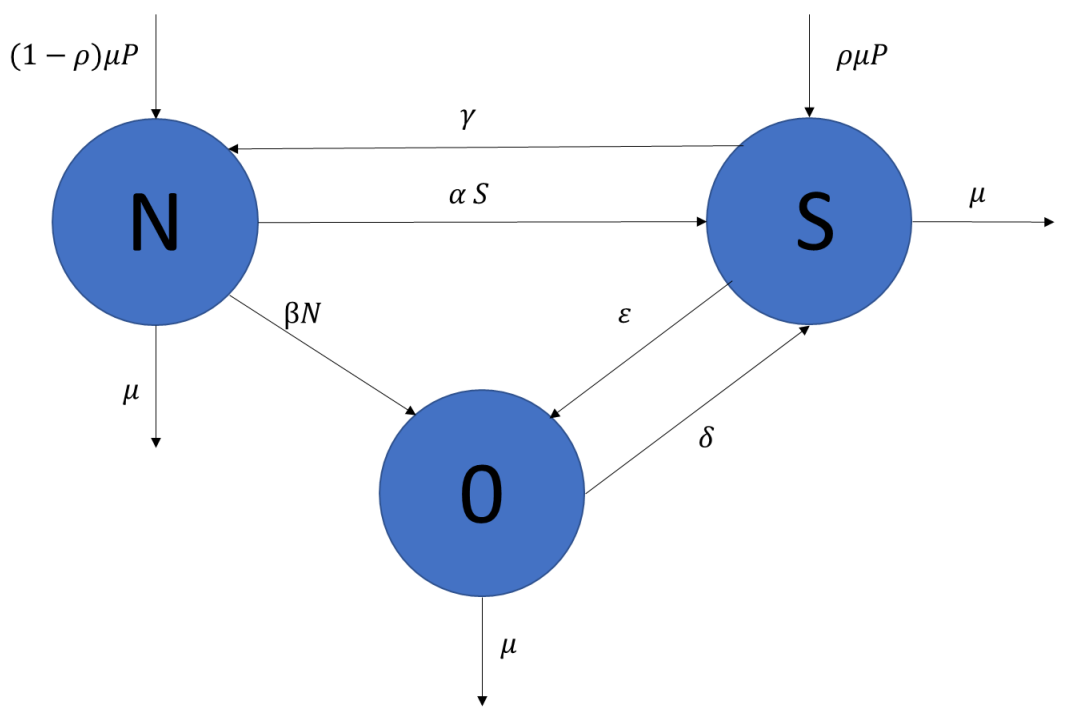

Figura 3.1: Modelo con control de la obesidad (2.1)

lo que en ecuaciones diferenciales ordinarias no lineales viene dado por

$$
\begin{aligned}
\frac{d N}{d t} & =(1-\rho) \mu P(t)-\alpha \frac{S(t)}{P(T)} N(t)-\beta \frac{O(t)}{P(t)} N(t)+\gamma S(t)-\mu N(t) \\
\frac{d S}{d t} & =\rho \mu P(t)+\alpha \frac{S(t)}{P(T)} N(t)+\delta O(t)-(\epsilon+\gamma+\mu) S(t) \\
\frac{d O}{d t} & =\beta \frac{O(t)}{P(t)} N(t)+\epsilon S(t)-(\delta+\mu) O(t)
\end{aligned}
$$


donde muchos de los parámetros son los del modelo (2.1) vistos en la (Tabla 2.2) y otros son estos:

\begin{tabular}{|c|c|}
\hline Parámetros CI & Significado \\
\hline$\gamma$ & $\begin{array}{l}\text { Tasa a la cual un individuo con sobrepeso adquiere un peso normal porque } \\
\text { entra en control de sobrepeso (dieta) }\end{array}$ \\
\hline$\delta$ & $\begin{array}{l}\text { Tasa a la cual un individuo con obesidad se convierte a individuo con sobrepeso } \\
\text { porque entra en control de obesidad (dieta) }\end{array}$ \\
\hline
\end{tabular}

Tabla 3.1: Valores de las condiciones iniciales (CI) del sistema (3.1)

3.1. Reducción del sistema. En el sistema (3.1), observamos que la población total $P(t)$ es constante, dado que

$$
\frac{d P}{d t}=\frac{d(N+S+O)}{d t}
$$

donde $P(t)=P($ cte), se tiene $N(t)=P-(S(t)+O(t))$. En consecuencia, el sistema (3.1) se reduce al estudio de un sistema bidimensional dado por

$$
\frac{d S}{d t}=\rho \mu P(t)+\alpha \frac{S(t)}{P}[P-(S(t)+O(t))]+\delta O(t)-(\epsilon+\gamma+\mu) S(t)
$$

$$
\frac{d O}{d t}=\beta \frac{O(t)}{P}[P-(S(t)+O(t))]+\epsilon S(t)-(\delta+\mu) O(t)
$$

3.2. Normalización del sistema. Normalizando el sistema (3.2), consideramos las subpoblaciones $s(t)=S(t) P$ у $o(t)=O(t) P$, debido a que la población total es constante, sin perdida de generalidad, se obtiene

$$
\frac{d s}{d t}=\rho \mu+\alpha s[1-(s+o)]+\delta o-(\epsilon+\gamma+\mu) s
$$

$$
\frac{d o}{d t}=\beta o[1-(s+o)]+\epsilon s-(\delta+\mu) o
$$

\subsection{Análisis del modelo. Existencia de las soluciones}

Teorema 5. Para todo $s_{0}, o_{0}>0$, existen $s, o:[0, \infty) \longrightarrow[0, \infty)$ que resuelven el sistema (3.3) con condiciones iniciales $s=s_{0}, o=o_{0}$.

Demostración: La existencia de las soluciones está garantizada por el teorema de Peano.

Teorema 6. Todas las soluciones del sistema (6) son acotadas y positivas.

Demostración: El conjunto de soluciones del sistema (6) se prueba que son acotadas y positivas con la condición de conjunto positivamente invariante; en el sentido que si las condiciones iniciales del sistema son acotadas y no negativas (región invariante), las soluciones permanecen en dicha región en toda su definición.

Punto de equilibrio libre de obesidad y tasas básicas de reemplazo. Con (2.3) ya reducido y normalizado al sistema (3.3), buscamos la solución constante que represente la situación de que una población quede libre de obesidad. Para hallar los puntos de equilibrio, resolvemos el sistema algebraico, indicado por las isoclinas

$$
\begin{aligned}
\rho \mu+\alpha s[1-(s+o)]+\delta o-(\epsilon+\gamma+\mu) s & =0 \\
\beta o[1-(s+o)]+\epsilon s-(\delta+\mu) o & =0
\end{aligned}
$$


Teorema 7. Si $\rho=0$, los puntos críticos se dividen en tres casos:

1. El punto libre de sobrepeso y obesidad $(s, o)=(0,0)$ existe si $\alpha=\epsilon+\gamma+\mu$

2. El punto libre de obesidad $(s, o)=\left(s^{*}, 0\right)$ existe si $\alpha>\epsilon+\gamma+\mu$.

3. El punto persistencia de sobrepeso y obesidad $(s, o)=\left(s^{* *}, o^{* *}\right)$ existe si $(\epsilon+\gamma+\mu-\alpha)(\beta-\alpha)>$ 0 , donde

$$
s^{* *}=(\alpha-\epsilon-\mu)\left(\frac{\mu(\beta-\alpha)+\epsilon \beta}{(\epsilon+\gamma+\mu)(\beta-\alpha)}\right)
$$

$y$

$$
o^{* *}=\left(\frac{\epsilon+\mu-\alpha}{\epsilon(\epsilon+\gamma+\mu)(\beta-\alpha)}\right)
$$

Las tasas básicas de reemplazo son aquellos parámetros de salida que determinan la ocurrencia o no de la eliminación de las poblaciones de individuos con sobrepeso y obesidad. Para su cálculo utilizamos la matriz de la próxima generación.

Teorema 8. Si $\rho=0$, el punto de equilibrio libre de sobrepeso y obesidad es local y asintóticamente estable si $\mathcal{R}=\frac{\mu}{\beta}<1$, es inestable si $\mathcal{R}=\frac{\mu}{\beta}>1$.

Demostración: Al aplicar la matriz de la próxima generación, los autovalores de la matriz de la próxima generación correspondiente son $\lambda_{1}=\frac{\alpha}{\epsilon+\gamma+\mu}$ y $\lambda_{2}=\frac{\beta}{\mu}$

. El punto de equilibrio libre de sobrepeso y obesidad existe si $\alpha=\epsilon+\gamma+\mu$, luego $\lambda_{1}=1$. En consecuencia, por el Teorema 2 de la Ref. 11, tenemos que si $\mathcal{R}=\frac{\mu}{\beta}<1$, el punto $(s, o)=(0,0)$ es local y asintóticamente estable y si $\mathcal{R}=\frac{\mu}{\beta}>1$, dicho punto es inestable.

3.4. Estimación de Parametros. Trabajaremos el caso de obesidad en niños, vamos a calcular algunos parámetros estimados para tiempo $t$ en semanas y otros los tomaremos de la literatura de investigación. Consideramos que todos los niños nacen con peso normal. El parámetro de tiempo medio que un niño se queda en el sistema dinámico es 3 años, es decir. 156 semanas, luego $\mu=1 / 156 \mathrm{sem}^{-1}$. El parámetro con el que los padres de un individuo (niño) toma conciencia de su sobrepeso con mayor interés en su nutrición viene dado por $\epsilon=k * 0,0089$, donde el valor de 0,0089 se estima usando el crecimiento semanal del peso medio de un niño registrado en [12], es decir el número de semanas que se toma un niño con mala alimentación en lograr sobrepeso. Un individuo con consumo de comida no sana se toma 1/0,0089 semanas para ir del peso normal a sobrepeso, entonces esto multiplicado por la tasa de éxito para regresar a peso normal viene dado por $\epsilon=0,0089 * 0,312=2,7768 * 10^{-1}$ semanas $^{-1}$. El parámetro que mide el éxito parcial de que un individuo obeso en dieta disminuya su IMC a sobrepeso, es estimado así $\delta=\epsilon * 0,146=k * 1,2994 * 10^{-3}$ semanas $^{-1}$ [8].

Hacemos un resumen de los valores de los parámetros antes discutidos.

\begin{tabular}{|c|c|}
\hline Parámetros & Valor \\
\hline$\rho$ & 0 \\
\hline$\mu$ & $\frac{1}{156}$ semanas $^{-1}$ \\
\hline$\alpha$ & 0,0089 semana $^{-1}$ \\
\hline$\beta$ & $\beta \in(0,1)$ \\
\hline$k$ & $k \in(0,1)$ \\
\hline$\epsilon$ & $k * 0,0089$ semanas $^{-1}$ \\
\hline$\gamma$ & $2,7768 * 10^{-3}$ semanas $^{-1}$ \\
\hline$\delta$ & $k * 1,2994 * 10^{-3}$ semanas $^{-1}$ \\
\hline
\end{tabular}

Tabla 3.2: Estimaciones de los valores de los parámetros del sistema (3.1)

Los parámetros $\beta$ y $k$ serán estimados de acuerdo con los resultados de la data con la que se trabaje. 
3.5. Simulaciones numéricas. Consideramos condiciones iniciales en el año $2007(t=0)$ y condiciones finales en el año $2010(t=156)$ para el modelo (3.1).

Con los valores de los parámetros obtenidos por literatura especializada y las condiciones iniciales, realizamos algunas simulaciones para diferentes valores de $\beta$ y $k$ tratando de encontrar valores adecuados para la realidad correspondiente.

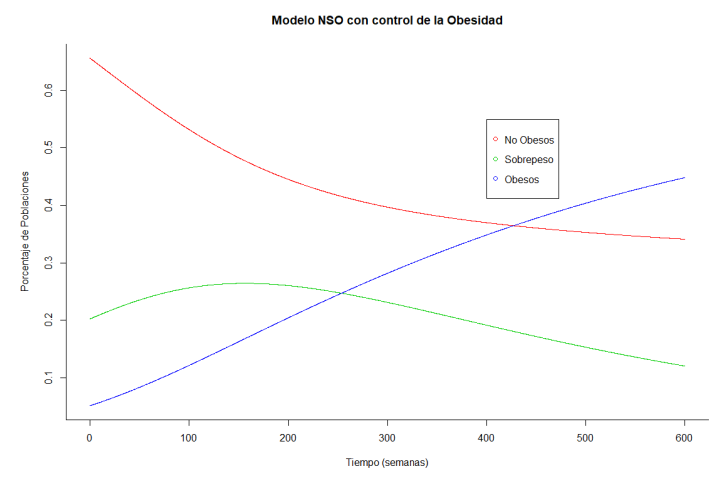

Figura 3.2: Modelo con control de la obesidad (3.1)

Hacemos una comparación gráfica de los modelos propuestos (1) y (4) y apreciamos el aporte del control sobre la dinámica de la obesidad.
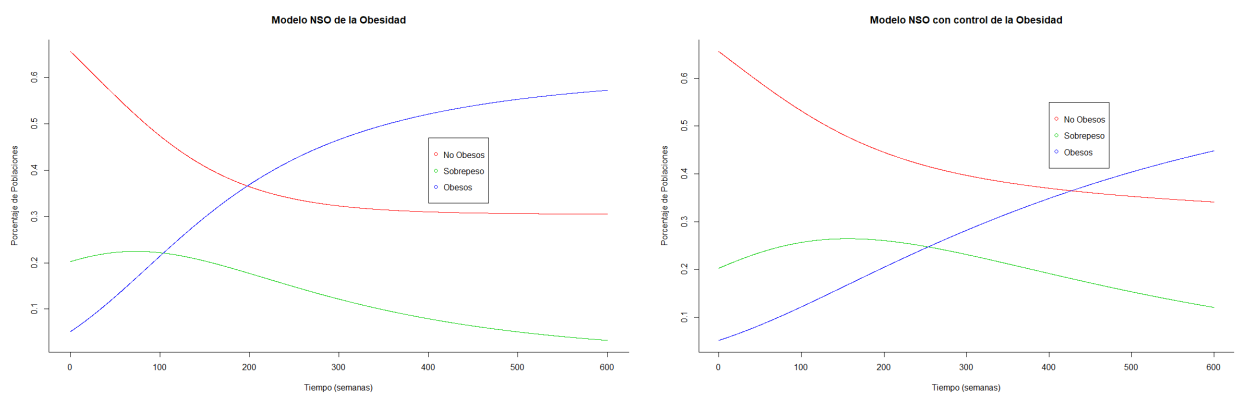

Figura 3.3: Comparación de los Modelos sin y con control de la obesidad (3.1)

4. Conclusiones. En este trabajo, analizamos dos modelos en ecuaciones diferenciales ordinarias que permiten analizar a la obesidad desde un punto de vista epidemiològico. El primero nos describe la propagaciòn de dos estados de anormalidad en peso de los individuos, sin considerar tratamiento alguno. El segundo modelo, coloca un parametro que podria describir la tasa de efectividad de un control que este caracterizado por un cambio saludable en el estilo de vida del paciente, cuando toma conciencia de su peso o sufre las consecuencias sobre otros aspectos de su salud. Esto puede suceder en dos etapas cuando el individuo pasa de su calidad de obeso a sobrepeso y el otro de sobrepeso a no obeso.

La pregunta que se presenta es que tan efectivo podria ser el control considerado y cual seria su relacion con la tasa de desercion y volver a los malos habitos de vida. En este trabajo hemos considerado parametros de control constantes. Nuestro análisis muestra que cuando $\mathcal{R}<1$ el punto de equilibrio libre de obesidad es local y asintoticamente estable y cuando $\mathcal{R}>1$ este es inestable.

Agradecimientos. La investigación fue financiada por el Instituto de Investigación Científica de la Universidad de Lima (IDIC-ULIMA). Proyecto 2019 Obesidad como epidemia: Predicciones a partir de modelos matemáticos.

ORCID and License

Roxana López Cruz https: / / orcid.org/0000-0002-7703-5784

This work is licensed under the Creative Commons Attribution-NoComercial-ShareAlike 4.0. 


\section{Referencias}

[1] Arenas A, González-Parra G, Jódar L. Periodic solutions of nonautonomous differential systems modeling obesity population. Chaos, Solitons \& Fractals. 2009; 42(2):1234-1244.

[2] Evangelista A, Ortiz A, Ríos-Soto K, Urdapilleta A. USA the fast food nation: Obesity as an epidemic. Los Alamos National Laboratory; 2004.

[3] Lozano-Ochoa E, Camacho J, Vargas-De-León C. Qualitative Stability Analysis of an Obesity Epidemic Model with Social Contagion. Discrete Dynamics in Nature and Society; 2017.

[4] Ministerio de Salud. Instituto Nacional de Estadística (INE). Encuesta Nacional de Nutrición y Salud (ENNSA 1984). Lima; 1984.

[5] Pajuelo R. La obesidad en el Perú. An Fac Med. 2017; 78(2):179-185.

[6] Obesity and Overweight. Fact Sheet, October 2017. Disponible en: http://www.who.int/me-diacentre/factsheets/fs311/en/. Accedido el 13 de octubre 2017, Intensive Care Medicine. 2017; 29(5):787-794.

[7] Thomas D, Weedermann M, Fuemmeler B, Martin C, Dhurandhar N, Bredlau C, Bouchard C. Dynamic model predicting overweight, obesity, and extreme obesity prevalence trends. Obesity. 2014; 22(2):590-597.

[8] Vásquez R. Estabilidad de un modelo matemático de la obesidad con factor genético-ambiental [internet]. Lima: UNMSM; 2018[citado 2 febero 2020]. Disponible en: https:// cybertesis . unmsm. edu.pe/handle/20.500.12672/ 8399 